\title{
THE GALAXY DISTRIBUTION SURROUNDING THE SCULPTOR VOID
}

\author{
GEORGE RHEE \\ New Mexico State University, Department of Astronomy, Box 30001/Department 4500, Las Cruces, New Mexico 88003 \\ Peter Katgert \\ Leiden Observatory, Postbus 9513, 2300 RA Leiden, The Netherlands \\ Received 13 September 1991; revised 21 January 1992
}

\begin{abstract}
We present the results of a survey of galaxy redshifts toward the Sculptor void. Radial velocities for 113 galaxies in the right ascension range $22^{\mathrm{h}}-2^{\mathrm{h}}$ and declination range $-35^{\circ}$ to $-25^{\circ}$ have been obtained. This region is of interest since there are suggestions that it contains a two-dimensional sheet of galaxies at the interface between two voids. We have added our redshifts to the known redshifts in this area and have applied a statistical test to determine the nature of the galaxy distribution in this volume of space. The method is aimed at quantifying the topology of the distribution. The method yields quantitative results which are consistent with a distribution of sheets and voids.
\end{abstract}

\section{INTRODUCTION}

In this paper, we present the results of a redshift survey we have made towards the Sculptor void. In recent years, redshift surveys have revealed large structures in the galaxy distribution with a variety of topologies. The galaxy distribution has been described as filamentary or cellular (Einasto $e t$ al. 1980; Batuski \& Burns 1985), sheetlike (Geller \& Huchra 1989), bubblelike (de Lapparent et al. 1986), or "spongelike" in appearance (Gott et al. 1986), or some combination of these (Haynes \& Giovanelli 1986; da Costa et al. 1988).

Observations of these large structures provide a powerful constraint on models of galaxy formation. The current, most popular model of galaxy formation has too little large scale power to account for a number of recent observations. One of the most troubling observations is the large coherence scale of peculiar velocity flows (Lynden-Bell et al. 1988), whose theoretical implications have been discussed by Kaiser (1988), Bertschinger \& Juszkiewicz (1988), and Ostriker \& Suto (1990). Further problematic observations include the high amplitude of the cluster-cluster correlation function (Bahcall \& Soneira 1983; Klypin \& Kopylov 1983), the angular correlation function of galaxies at large separations (Maddox et al. 1990), and the existence of large structures like the Perseus Pisces supercluster (Haynes \& Giovanelli 1986, 1988), the Bootes void (Kirshner et al. 1987), and the "great wall" that dominates the northern sky in the extended CfA survey (Geller \& Huchra 1989). Prior to the discovery of the great wall, the evidence for sheetlike structures in the galaxy distribution was circumstantial, since in the CfA slice redshift, surveys filamentary structures were observed. We thus decided to search for a sheetlike structure with a view to mapping it and providing direct evidence for sheets. We made a redshift survey in a $7^{\circ} \times 6^{\circ}$ area in a region where a large surface density of galaxies is present on the sky survey, and combine our data with data from the literature in a much larger area of $10^{\circ} \times 60^{\circ}$ centered on our smaller area.

Parker et al. (1987), using spectra from low dispersion UKST objective prism plates, have used the $4000 \AA$ feature, when detectable, to measure galaxy redshifts to an accuracy of 0.01 in $z$. They scanned a $5.35^{\circ} \times 5.35^{\circ}$ field centered on $00^{\mathrm{h}} 00^{\mathrm{m}},-35^{\circ} 00^{\prime}$. They found evidence for a filamentary structure extended in declination and a low redshift en- hancement extended perpendicular to the filament. This enhancement appeared sheetlike in projection, and we decided to further investigate it as a candidate for a sheet in redshift. While our survey was under way, Maurellis et al. (1990) published a study of this area based on redshifts compiled from the literature. They found evidence for a wall of galaxies $650 \mathrm{~km} / \mathrm{s}$ thick $2000 \times 2000 \mathrm{~km} / \mathrm{s}$ wide, forming the interface to the Sculptor and Eridanus voids.

\section{THE DATA}

\subsection{Observations}

In order to further investigate this interesting region of the sky, we have measured radial velocities of 113 galaxies in the right ascension range $22^{\mathrm{h}}-2^{\mathrm{h}}$ and declination range $-35^{\circ}-$ $-25^{\circ}$. We used the European Southern Observatory $1.5 \mathrm{~m}$ telescope. Two runs were made in 1988 August and 1989 August, respectively. We used a GEC chip with a readout noise of $12 \mathrm{e}$ with a Boller and Chivens spectrograph at the Cassegrain focus of the $1.5 \mathrm{~m}$ telescope. The spectra we obtained had $7 \AA$ resolution. Initially, candidate galaxies for spectroscopy were selected by eye using film copies of the SERC IIIa-J Sky Survey. By the time our second run was due, the Surface Photometry Catalog of the ESO-Uppsala galaxies had been published and we used it to sheet candidates for spectroscopy. To narrow down the list of galaxies, we selected galaxies with $B_{T}<15.0 . B_{T}$ is the total blue magnitude measured to $\mu_{B} \simeq 25-26$ [see Lauberts \& Valentijn (1989) for the exact definition]. In total, we have obtained radial velocities for 113 galaxies, 8 of which are not in the $L V$ catalog. Radial velocities were measured using the crosscorrelation method of Tonry \& Davis (1979) (see also Rhee \& Katgert 1988). We used a spectrum of M32 as a template. The spectrum was obtained from Keel (1983), it was taken with an 8 " aperture using the image dissector scanner on the $1 \mathrm{~m}$ Nickel telescope at Lick Observatory. The data are listed in Table 1. We list the LV catalog number, right ascension, declination heliocentric radial velocity total $B$ and $R$ magnitudes.

In Table 2 we compare our radial velocities with previously measured radial velocities. The velocities are taken from two sources. We searched the LV (1989) catalog and the catalog of da Costa et al. (1991) for galaxies with previously measured redshifts. There are 8 galaxies in the $\mathrm{LV}$ catalog 
TABLE 1. The sample.

\begin{tabular}{|c|c|c|c|c|c|c|c|c|c|}
\hline \multirow[t]{2}{*}{ Object } & \multicolumn{3}{|c|}{$\alpha \quad(1950)$} & \multicolumn{2}{|c|}{$\delta \quad(1950)$} & \multirow[t]{2}{*}{$v$} & \multirow[t]{2}{*}{$\sigma_{v}$} & \multirow[t]{2}{*}{$\mathrm{B}_{T}$} & \multirow[t]{2}{*}{$\mathrm{R}_{T}$} \\
\hline & $\mathrm{H}$ & $\mathrm{M}$ & $\mathrm{S}$ & $\circ$ & M & & & & \\
\hline 4090100 & 0 & 1 & 44 & -30 & 10.1 & 19398 & 135 & 15.25 & 14.52 \\
\hline 4090130 & 0 & 2 & 27 & -30 & 47.1 & 8154 & 114 & 15.00 & 13.62 \\
\hline 4090140 & 0 & 2 & 58 & -30 & 51.9 & 8495 & 112 & 15.28 & 14.10 \\
\hline 4720180 & 0 & 9 & 42 & -27 & 28.0 & 10383 & 100 & 14.99 & 13.71 \\
\hline 4732100 & 0 & 16 & 28 & -23 & 09.5 & 7393 & 91 & 14.65 & 13.72 \\
\hline 4730250 & 0 & 29 & 21 & -26 & 59.8 & 7266 & 133 & 14.68 & 13.05 \\
\hline 4740070 & 0 & 35 & 21 & -26 & 55.4 & 5649 & 167 & 14.81 & 13.56 \\
\hline 4740270 & 0 & 44 & 44 & -26 & 39.3 & 5645 & 202 & 14.88 & 13.58 \\
\hline 2950060 & 0 & 45 & 58 & -38 & 30.4 & 4948 & 102 & 14.42 & 13.17 \\
\hline 3510110 & 0 & 48 & 40 & -32 & 41.6 & 9657 & 100 & 14.69 & 13.05 \\
\hline 4120010 & 0 & 57 & 50 & -31 & 4.8 & 9711 & 263 & 14.72 & 13.69 \\
\hline 4120070 & 1 & 6 & 24 & -28 & 50.9 & 5012 & 131 & 13.50 & 11.71 \\
\hline 4120140 & 1 & 9 & 59 & -32 & 19.6 & 9201 & 111 & 15.06 & 13.69 \\
\hline 4120160 & 1 & 10 & 36 & -31 & 42.9 & 5483 & 120 & 14.57 & 13.22 \\
\hline 3520220 & 1 & 11 & 24 & -34 & 10.7 & 10025 & 285 & 15.12 & 14.05 \\
\hline 3520241 & 1 & 11 & 38 & -32 & 54.6 & 10016 & 245 & 15.29 & 14.55 \\
\hline 4750150 & 1 & 13 & 23 & -27 & 6.4 & 3531 & 180 & 14.08 & 12.67 \\
\hline 3520550 & 1 & 19 & 14 & -33 & 25.1 & 10808 & 121 & 14.28 & 12.99 \\
\hline 3520570 & 1 & 19 & 44 & -34 & 27.5 & 5600 & 141 & 14.63 & 13.39 \\
\hline 4130050 & 1 & 23 & 18 & -30 & 37.5 & 9150 & 60 & 14.17 & 12.89 \\
\hline 4760180 & 1 & 28 & 54 & -27 & 7.0 & 5775 & 153 & 14.49 & 13.03 \\
\hline 3530290 & 1 & 36 & 13 & -33 & 51.7 & 8911 & 47 & 14.75 & 13.58 \\
\hline 4130190 & 1 & 38 & 3 & -29 & 10.0 & 5618 & 126 & 13.71 & 12.60 \\
\hline 3530310 & 1 & 38 & 21 & -33 & 52.4 & 8670 & 102 & 14.98 & 13.45 \\
\hline 4130200 & 1 & 38 & 23 & -32 & 4.4 & 5979 & 122 & 14.05 & 13.28 \\
\hline 4140010 & 1 & 43 & 37 & -20 & 17.3 & 5779 & 74 & 14.82 & 13.18 \\
\hline 4770070 & 1 & 47 & 6 & -26 & 59.6 & 5898 & 177 & 14.85 & 13.42 \\
\hline 4770090 & 1 & 47 & 33 & -20 & 31.9 & 9537 & 157 & 14.76 & 13.76 \\
\hline 4140050 & 1 & 47 & 34 & $-2 \pi$ & 42.8 & 4134 & 87 & 14.56 & 13.05 \\
\hline 4770140 & 1 & 52 & 42 & -26 & 15.8 & 8827 & 81 & 14.52 & 12.77 \\
\hline 4140200 & 1 & 56 & 55 & -28 & 3.1 & 4473 & 220 & 14.70 & 13.49 \\
\hline 4770200 & 1 & 58 & 18 & -25 & 17.6 & 4181 & 114 & 14.71 & 13.22 \\
\hline 4770210 & 1 & 58 & 32 & -25 & 16.1 & 4415 & 88 & 14.07 & 12.49 \\
\hline 4770220 & 1 & 59 & 13 & -25 & 9.9 & 22815 & 160 & 14.52 & 13.03 \\
\hline 4140270 & 2 & 02 & 29 & -29 & 32.5 & 5080 & 181 & 14.46 & 13.03 \\
\hline
\end{tabular}

TABLE 1. (continued)

\begin{tabular}{|c|c|c|c|c|c|c|c|c|c|}
\hline \multirow[t]{2}{*}{ Object } & \multicolumn{3}{|c|}{$\alpha \quad(1950)$} & \multicolumn{2}{|c|}{$\delta \quad(1950)$} & \multirow[t]{2}{*}{$v$} & \multirow[t]{2}{*}{$\sigma_{v}$} & \multirow[t]{2}{*}{$\mathrm{B}_{T}$} & \multirow[t]{2}{*}{$\mathrm{R}_{T}$} \\
\hline & $\mathrm{H}$ & $\mathbf{M}$ & $\mathrm{S}$ & $\bullet$ & $\mathrm{M}$ & & & & \\
\hline 4670081 & 22 & 4 & 2 & -31 & 18.0 & 9772 & 94 & 14.93 & 13.94 \\
\hline 5320210 & 22 & 5 & 25 & -25 & 18.4 & 5553 & 203 & 14.65 & 13.47 \\
\hline 5320260 & 22 & 8 & 5 & -25 & 19.2 & 5042 & 190 & 14.71 & 13.25 \\
\hline 4670240 & 22 & 11 & 12 & -29 & 37.9 & 4498 & 222 & 13.31 & 12.49 \\
\hline 4670300 & 22 & 12 & 5 & -28 & 10.9 & 4039 & 99 & 14.37 & 13.31 \\
\hline 5330070 & 22 & 13 & 46 & -27 & 4.2 & 8214 & 88 & 14.34 & 13.32 \\
\hline 4670420 & 22 & 16 & 25 & -28 & 39.2 & 8485 & 235 & 14.28 & 13.26 \\
\hline 5330140 & 22 & 17 & 2 & -26 & 35.6 & 2636 & 142 & 13.75 & 12.95 \\
\hline 4050090 & 22 & 17 & 34 & -32 & 56.3 & 16911 & 183 & 14.87 & 13.79 \\
\hline 5330180 & 22 & 19 & 31 & -27 & 8.1 & 5749 & 162 & 14.86 & 13.64 \\
\hline 4670460 & 22 & 19 & 51 & -32 & 27.1 & 8341 & 85 & 14.60 & 13.13 \\
\hline 5330240 & 22 & 20 & 55 & -26 & 52.0 & 7582 & 70 & 14.19 & 13.13 \\
\hline 4670580 & 22 & 23 & 16 & -31 & 7.4 & 8541 & 86 & 14.86 & 13.44 \\
\hline 4670620 & 22 & 24 & 4 & -31 & 8.5 & 33696 & 157 & 14.94 & 14.44 \\
\hline 4670650 & 22 & 24 & 54 & -30 & 17.7 & 8461 & 133 & 14.64 & 13.96 \\
\hline 5330350 & 22 & 27 & 21 & -27 & 1.8 & 10002 & 92 & 14.68 & 13.30 \\
\hline 4680130 & 22 & 30 & 57 & -27 & 30.2 & 6887 & 81 & 14.34 & 13.71 \\
\hline 4050290 & 22 & 31 & 13 & -32 & 39.3 & 23383 & 26 & 14.61 & 14.19 \\
\hline 4680160 & 22 & 33 & 21 & -31 & 59.2 & 8016 & 85 & 14.67 & 13.66 \\
\hline 5340100 & 22 & 36 & 06 & -25 & 58.2 & 3390 & 173 & 13.90 & 12.11 \\
\hline 4060100 & 22 & 42 & 4 & -34 & 28.5 & 8791 & 130 & 14.97 & 14.76 \\
\hline 4060370 & 22 & 56 & 22 & -34 & 20.6 & 8964 & 62 & 14.99 & 13.94 \\
\hline 4070020 & 23 & 1 & 56 & -34 & 19.6 & 1727 & 170 & 14.08 & 12.84 \\
\hline 4690120 & 23 & 4 & 24 & -28 & 53.0 & 9678 & 97 & 15.28 & 14.10 \\
\hline 4700020 & 23 & 19 & 53 & -29 & 33.3 & 6879 & 97 & 14.70 & 13.23 \\
\hline RK105 & 23 & 22 & 28 & -31 & 25.9 & 10668 & 102 & & \\
\hline 4700110 & 23 & 26 & 56 & -29 & 6.4 & 7244 & 117 & 14.91 & 13.55 \\
\hline RK99 & 23 & 33 & 37 & -31 & 52.7 & 14871 & 112 & & \\
\hline 4710020 & 23 & 38 & 17 & -28 & 37.0 & 8339 & 160 & 14.51 & 0.00 \\
\hline RK112 & 23 & 38 & 52 & -29 & 35.9 & 15685 & 110 & & \\
\hline RK113 & 23 & 38 & 58 & -29 & 30.8 & 15209 & 130 & & \\
\hline RK115 & 23 & 39 & 14 & -28 & 18.1 & 8599 & 180 & & \\
\hline RK111 & 23 & 39 & 39 & -30 & 28.5 & 18936 & 150 & & \\
\hline 4710070 & 23 & 41 & 48 & -31 & 57.5 & 11099 & 100 & 15.19 & 13.94 \\
\hline 4080240 & 23 & 42 & 2 & -34 & 46.0 & 11745 & 90 & 15.20 & 13.90 \\
\hline
\end{tabular}

TABLE 1. (continued)

\begin{tabular}{|c|c|c|c|c|c|c|c|c|c|}
\hline \multirow[t]{2}{*}{ Object } & \multicolumn{3}{|c|}{$\alpha \quad(1950)$} & \multicolumn{2}{|c|}{$\delta \quad(1950)$} & \multirow[t]{2}{*}{$v$} & \multirow[t]{2}{*}{$\sigma_{v}$} & \multirow[t]{2}{*}{$\mathrm{B}_{T}$} & \multirow[t]{2}{*}{$\mathrm{R}_{\mathrm{T}}$} \\
\hline & $\mathrm{H}$ & $\mathbf{M}$ & $\mathrm{S}$ & $\circ$ & M & & & & \\
\hline RK108 & 23 & 42 & 17 & -32 & 21.3 & 18733 & 220 & & \\
\hline 4710090 & 23 & 43 & 11 & -29 & 47.7 & 10494 & 98 & 13.85 & 12.38 \\
\hline 4710110 & 23 & 43 & 47 & -28 & 17.0 & 8418 & 140 & 15.24 & 13.85 \\
\hline 4710111 & 23 & 43 & 47 & -28 & 16.8 & 8493 & 116 & 14.77 & 13.53 \\
\hline 4710120 & 23 & 44 & 00 & -29 & 20.9 & 10466 & 90 & 14.50 & 13.06 \\
\hline 4710130 & 23 & 44 & 02 & -28 & 59.2 & 19073 & 190 & 15.60 & 14.09 \\
\hline RK102 & 23 & 44 & 24 & -29 & 26.9 & 10251 & 20 & & \\
\hline 4710140 & 23 & 44 & 39 & -28 & 14.1 & 8479 & 70 & 14.69 & 13.40 \\
\hline 4710170 & 23 & 44 & 53 & -28 & 23.2 & 8224 & 152 & 14.03 & 12.55 \\
\hline 4710180 & 23 & 45 & 9 & -27 & 45.9 & 8873 & 156 & 14.94 & 13.84 \\
\hline 4710190 & 23 & 45 & 9 & -28 & 25.0 & 8623 & 90 & 13.42 & 11.99 \\
\hline RK94 & 23 & 45 & 09 & -28 & 24.9 & 8070 & 120 & & \\
\hline 4710200 & 23 & 45 & 15 & -30 & 48.0 & 2984 & 125 & .12 .10 & 11.11 \\
\hline 4710220 & 23 & 46 & 39 & -30 & 31.5 & 13747 & 70 & 14.65 & 13.43 \\
\hline 4710230 & 23 & 46 & 40 & -29 & 18.5 & 10569 & 165 & 14.97 & 13.78 \\
\hline 4710240 & 23 & 47 & 16 & -28 & 13.7 & 8925 & 60 & 14.74 & 13.36 \\
\hline RK12 & 23 & 48 & 03 & -28 & 42.7 & 8698 & 111 & & \\
\hline RK33 & 23 & 48 & 34 & -31 & 37.0 & 13489 & 140 & & \\
\hline RK6 & 23 & 49 & 19 & -28 & 11.5 & 8835 & 112 & & \\
\hline 4710280 & 23 & 49 & 20 & -30 & 52.5 & 14896 & 130 & 14.88 & 13.95 \\
\hline RK17 & 23 & 49 & 35 & -29 & 21.5 & 8770 & 90 & & \\
\hline 4710320 & 23 & 49 & 47 & -30 & 30.5 & 8660 & 73 & 14.74 & 13.58 \\
\hline RK16 & 23 & 49 & 49 & -29 & 18.2 & 8716 & 105 & & \\
\hline RK23 & 23 & 49 & 50 & -29 & 54.5 & 13016 & 130 & & \\
\hline 4710340 & 23 & 49 & 56 & -30 & 27.5 & 8877 & 107 & 14.33 & 13.20 \\
\hline RK10 & 23 & 50 & 09 & -28 & 50.9 & 17703 & 200 & & \\
\hline 4710360 & 23 & 50 & 37 & -32 & 0.0 & 14425 & 108 & 15.17 & 13.76 \\
\hline RK21 & 23 & 50 & 50 & -29 & 48.9 & 8753 & 120 & & \\
\hline 4710390 & 23 & 51 & 51 & -29 & 9.9 & 8683 & 80 & 15.29 & 13.95 \\
\hline RK27 & 23 & 53 & 15 & -30 & 08.8 & 8655 & 152 & & \\
\hline 4710450 & 23 & 53 & 49 & -31 & 37.9 & 8695 & 130 & 14.18 & 12.81 \\
\hline 4710471 & 23 & 53 & 51 & -29 & 18.1 & 8909 & 130 & 14.69 & 13.33 \\
\hline 4710480 & 23 & 54 & 22 & -32 & 24.7 & 8867 & 200 & 14.96 & 14.19 \\
\hline 4710500 & 23 & 55 & 25 & -30 & 8.7 & 8899 & 85 & 14.84 & 13.53 \\
\hline RK45 & 23 & 55 & 20 & -32 & 05.6 & 8653 & 95 & & \\
\hline
\end{tabular}

TABle 1. (continued)

\begin{tabular}{|c|c|c|c|c|c|c|c|c|c|}
\hline \multirow[t]{2}{*}{ Object } & \multicolumn{3}{|c|}{$\alpha \quad(1950)$} & \multirow[t]{2}{*}{$\delta$} & $(1950)$ & \multirow[t]{2}{*}{$v$} & \multirow[t]{2}{*}{$\sigma_{v}$} & \multirow[t]{2}{*}{$\mathrm{B}_{T}$} & \multirow[t]{2}{*}{$\mathrm{R}_{T}$} \\
\hline & $\mathrm{H}$ & $\mathrm{M}$ & $\mathrm{S}$ & & M & & & & \\
\hline 4710510 & 23 & 55 & 43 & -30 & 23.3 & 8988 & 75 & 14.83 & 13.82 \\
\hline RK52 & 23 & 56 & 05 & -30 & 07.3 & 8827 & 70 & & \\
\hline 4710540 & 23 & 56 & 45 & -32 & 0.6 & 8360 & 66 & 14.75 & 13.78 \\
\hline 4090010 & 23 & 58 & 45 & -27 & 41.8 & 8168 & 116 & 14.92 & 13.42 \\
\hline 4090070 & 23 & 59 & 59 & -30 & 54.0 & 8971 & 80 & 14.50 & 13.20 \\
\hline
\end{tabular}


TABLE 2. Previously measured redshifts.

\begin{tabular}{|c|c|c|c|c|c|c|c|c|c|}
\hline \multirow[t]{2}{*}{ Object } & \multicolumn{3}{|c|}{$\alpha \quad(1950)$} & \multirow[t]{2}{*}{$\delta$} & (1950) & \multirow[t]{2}{*}{$v_{R K}$} & \multirow[t]{2}{*}{$\sigma_{v R K}$} & \multirow[t]{2}{*}{$v_{L V}$} & \multirow[t]{2}{*}{$\sigma_{v L V}$} \\
\hline & $\mathrm{H}$ & $\mathrm{M}$ & $\mathrm{S}$ & & M & & & & \\
\hline 4710090 & 23 & 43 & 11 & -29 & 47.7 & 10494 & 98 & 10380 & 100 \\
\hline 4710100 & 23 & 43 & 40 & -28 & 22.6 & 8418 & 140 & 8392 & 10 \\
\hline 4710140 & 23 & 44 & 39 & -28 & 14.1 & 8479 & 70 & 8560 & 14 \\
\hline 4710170 & 23 & 44 & 53 & -28 & 23.2 & 8224 & 152 & 8272 & 10 \\
\hline 4710190 & 23 & 45 & 09 & -28 & 25.0 & 8662 & 90 & 8631 & 10 \\
\hline 4710200 & 23 & 45 & 15 & -30 & 48.0 & 2984 & 125 & 2968 & 8 \\
\hline 4710220 & 23 & 46 & 39 & -30 & 31.5 & 13747 & 70 & 13809 & 33 \\
\hline 4710510 & 23 & 55 & 43 & -30 & 23.3 & 8988 & 75 & 9086 & 25 \\
\hline 4739250 & 00 & 29 & 21 & -26 & 59.8 & 7266 & 133 & 7235 & 45 \\
\hline 5320260 & 22 & 08 & 05 & -25 & 19.2 & 5042 & 190 & 4892 & 29 \\
\hline 4670240 & 22 & 11 & 12 & -29 & 37.9 & 4498 & 222 & 4303 & 15 \\
\hline 4670300 & 22 & 12 & 05 & -28 & 10.9 & 4039 & 99 & 4062 & 21 \\
\hline 4690120 & 23 & 04 & 24 & -28 & 53.0 & 9678 & 97 & 9701 & 35 \\
\hline 4710020 & 23 & 38 & 17 & -28 & 37.0 & 8339 & 160 & 8418 & 38 \\
\hline 4710090 & 23 & 43 & 11 & -29 & 47.7 & 10494 & 98 & 10380 & 20 \\
\hline
\end{tabular}

and 7 galaxies in the da Costa et al. catalog for which we have determined radial velocities. For the $8 \mathrm{LV}$ galaxies, we find that the mean difference between our measured redshifts and the $L V$ redshifts is $-13 \mathrm{~km} / \mathrm{s}$. The rms difference is $68 \mathrm{~km} /$ s. For the 7 galaxies from the da Costa catalog, we have a mean difference of $52 \mathrm{~km} / \mathrm{s}$ and a rms difference of $108 \mathrm{~km} /$ $\mathrm{s}$. For the joint sample of 15 galaxies, we find a mean difference of $17 \mathrm{~km} / \mathrm{s}$ and a rms difference of $89 \mathrm{~km} / \mathrm{s}$. These results are consistent with our error estimates for the redshifts of these galaxies, which vary between 70 and $152 \mathrm{~km} / \mathrm{s}$ with a mean of $102 \mathrm{~km} / \mathrm{s}$.

\subsection{The Database}

The goal of this project is to characterize the galaxy distribution surrounding the Sculptor void. In order to produce as complete a database as possible for studying the distribution of galaxies, we have combined the redshifts we obtained with those available from the literature. The two sources we used are the ESO-UPPSALA catalog and the compilation of Fairall (1988). We also searched the Abell et al. (1989) catalog for the presence of rich clusters in the surveyed volume. In total, we have 465 galaxies in the surveyed volume and 3 rich Abell clusters with measured redshifts.

Figures 1 and 2 display the data we obtained, combined with already available data. The dataset we examine is $10^{\circ}$ slice centered on $\delta=-30$, spanning the right ascension range $22^{\mathrm{h}}-2^{\mathrm{h}}$, and radial velocity range $0-15000 \mathrm{~km} / \mathrm{s}$. Figure 1 shows a wedge plot of the data. Figure 2 shows an equal area projection map of the galaxies shown in Fig. 1. Adopting the void nomenclature of Maurrelis et al. (1990), we see in Fig. 1 the Sculptor void extending from 3000 to $\sim 9000$ $\mathrm{km} / \mathrm{s}$. At $2000 \mathrm{~km} / \mathrm{s}$, we have the Eridanus void which borders on the Sculptor void.

\subsection{Completeness of Sample}

Ideally, when undertaking a redshift survey, one would like to take a galaxy photometric catalog and measure radial velocities of galaxies complete to a given magnitude limit. Projects using dedicated $4 \mathrm{~m}$ class telescopes with multifiber spectrographs are being initiated both in the northern and southern hemispheres. In view of the lack of a published photometric catalog in the southern sky, we have selected galaxies from the Lauberts-Valentijn catalog (1989), which contains all galaxies on the ESO quick blue survey plates

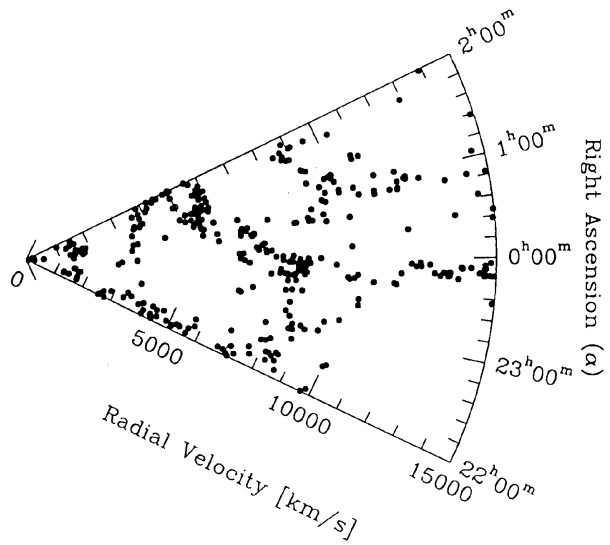

FIG. 1. Wedge plot of the galaxy distribution in our survey. The right ascension range is $22^{\mathrm{h}}<\alpha<2^{\mathrm{h}}$, the declination range $-35<\delta<-25$, and the redshift range is $0<z<15000 \mathrm{~km} / \mathrm{s}$.

with a maximum angular diameter of $1^{\prime}$. Since this is the only published galaxy catalog in the southern sky, we describe the completeness of our survey in terms of this catalog. The number of galaxies from the Lauberts-Valentijn catalog in the region $22^{\mathrm{h}} \leqslant \alpha \leqslant 2^{\mathrm{h}} ;-35^{\circ} \leqslant \delta \leqslant-25^{\circ}$ is 738 . With a limited amount of access to national facilities, we were not able to measure the radial velocities of all the galaxies in the LV catalog in this region. We find that to a $B_{\mathrm{T}}$ magnitude of 15.0 the survey is complete to $80 \%$. To the LV data, we have added galaxies from the literature with measured redshifts falling in the relevant right ascension and declination range. The main source from which we have added data is the compilation of Fairall (1988). Maurellis et al. suggest that redshift measurements are susceptible to selection effects in direction not in redshift. They have compared the sky coverage of the Fairall (1988) compilation with that of the LV catalog. By this, they mean that the ratio of Fairall galaxies to LV galaxies does not vary greatly from one ESO Schmidt plate field to another. We thus conclude that we are reasonably complete to a magnitude of $B_{\mathrm{T}}$ of 15.0 in the $\mathrm{LV}$ sample. We also conclude that our adding galaxies from the literature compilation of Fairall has not introduced a significant bias in our data, since the sky coverage of this latter sample is similar to that of the LV sample.

\section{ANALYSIS}

We have applied the topology method to characterize the galaxy distribution surrounding the Eridanus void. This method provides a quantitative description of the topology of the galaxy distribution. The method is based on an analy-

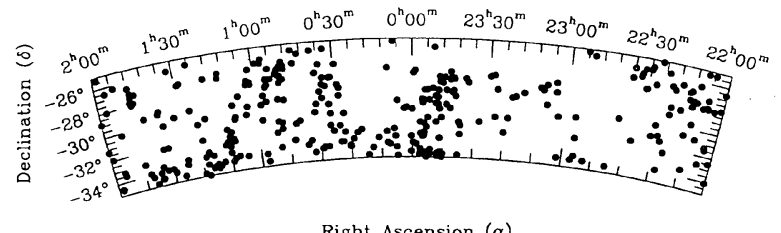

FIG. 2. Equal area projection of the galaxies in the sample. 
sis of the density contour surfaces of the smoothed density distribution (Gott et al. 1986).

Gott et al. (1986) have developed a method for quantifying the topology of the galaxy distribution. The method has been described in a clear manner by Melott (1990) and Gott (1988). In particular, Melott's review is accompanied by a number of figures that greatly help clarify the concepts of topology. We summarize the salient features of the method below. The goal of the method is to characterize the topology of the galaxy distribution revealed by redshift surveys. The first step is to smooth the data (points in $\alpha, \delta, v_{r}$ space) to produce a density field. A contour at constant density of this field produces a two-dimensional surface. This surface may have various topological characteristics. For example, it may be spongelike, in which case the matter and voids are interlocking and the surface displays a high degree of connectivity. At the other extreme, the surface may consist of a number of isolated lumps in an empty background, in which case it has a low degree of connectivity. This latter case is referred to by Melott (1990) as a meatball topology. Gott et al. (1986) have shown that one can quantify the topology of a surface using one number; the genus. The genus of an arbitrary surface with holes and disconnected pieces is: $g=$ number of holes - number of isolated regions. By holes we do not mean voids in the cosmological sense but rather in a topological sense, an example of which is the hole in a doughnut. An algorithm has been developed for estimating $g$ for a given density contour (Weinberg 1988). The algorithm is based on the Gauss-Bonnet theorem, which relates the gaussian curvature of a surface to the number of holes in the surface. The conventional wisdom in cosmology is based on the assumption that the structure we see in the galaxy distribution today was produced by the growth after recombination of random phase Gaussian fluctuations with a power spectrum $P(k)$. In the linear regime, fluctuations grow in place, simply increasing in amplitude, and the density contour surfaces as a function of $v$ (the number of standard deviations by which the contour threshold density is above or below the average density) do not change. Hamilton et al. (1986) give an analytical expression for the genus per unit volume as a function of $v$. A key result is that while the amplitude of the genus curve depends on the power spectrum $P(k)$, the shape of the curve is independent of the power spectrum. The analytical result shows that the $v=0$ contour is always spongelike, while for $|v|>1$ isolated clusters and voids are seen depending on whether $v$ is positive or negative.

One can then compare the observed genus curve from the redshift surveys with that expected on the basis of Gaussian fluctuations. A bubblelike distribution of galaxies would produce a genus curve $g(v)$ shifted to lower $v$ relative to the predicted curve for Gaussian fluctuations. This would indicate an excess of isolated regions at low $v$ and connected contours at high $v$.

As explained in Sec. 2, the data we have obtained are redshifts measured in a slice. Melott et al. (1989) have shown how the genus method can be extended to analyze a galaxy distribution sampled in two dimensions. Park et al. (1991) have applied this method to the slice data of de Lapparent $e t$ al. (1986) and Karachentsev \& Kopylov (1990).

To deal with two-dimensional distributions, the genus method must be generalized from three to two dimensions and a subtle point arises. In three dimensions, we can imagine either isolated clusters in a low density background, isolated voids in a high density background, or the third symmetric alternative of a sponge topology. In two dimensions, a closed curve necessarily divides space into an inside and outside and the sponge alternative does not exist. Melott et al. have shown that one can extend the genus method to two dimensions by referring to isolated high- and low-density regions, rather than voids and holes. $g(v)$ is now defined as the number of isolated high density regions - number of low density regions. A consequence of this definition of $g(v)$ is that the genus curve for a two-dimensional gaussian field is antisymmetric. As the density threshold is raised from the mean, the number of separate high-density regions will, at first, increase. As the density threshold is lowered from the mean, the number of isolated low-density regions will increase. For a Gaussian random density field, the mean twodimensional genus per unit area is:

$$
g(v)=\frac{1}{(2 \pi)^{3 / 2}} \frac{\left\langle k^{2}\right\rangle}{2} v e^{-v^{2} / 2},
$$

where $v$ is the threshold level in units of the standard deviation of the density fluctuations and $\left\langle k^{2}\right\rangle$ is the mean square of the wave number for a given power spectrum $P\left(k_{x}, k_{y}\right)$.

Clearly the two-dimensional method is less powerful than the three-dimensional approach in discriminating between various topologies. However, Melott et al. have shown, by comparing the analytic formula with the results of toy models and $N$-body simulations, that the method can yield useful results. By measuring the $g(v)$ curve from the data, one can see if the analytical form shown above is valid. If the curve deviates from the form shown above, one can, by comparing with simple models, infer how the observed galaxy distribution deviates from the Gaussian model.

We have applied the method described above to our data. The data must first be smoothed before the genus contours can be determined. We have chosen a smoothing radius of $650 \mathrm{~km} / \mathrm{s}$ and a maximum radius of $15000 \mathrm{~km} / \mathrm{s}$ for the sample. The smoothing radius is determined by the requirements that it be larger than the mean galaxy separation at $r_{\min }$ and $r_{\max }$ (where these minimum and maximum distances correspond to the limits of the survey) and that it maximize the number of independent resolution elements.

To estimate errors, 12 samples were generated from the original data using the bootstrap method described by Barrow et al. (1984). The bootstrap method involves generating pseudo-datasets from the original dataset. To generate a pseudo dataset, one labels the galaxies in the original sample 1 to $N$. One then generates a set of $N$ numbers randomly chosen between 1 and $N$. The $N$ random numbers indicate which galaxies are selected to be in the pseudo-dataset. The net result is that some galaxies in the original set are selected more than once while some are completely omitted. Strong structures consisting of many galaxies will not be affected much, while weak structures will often change.

Twelve samples were generated from the original sample in this manner. Using these 12 samples, the mean genus and $1 \sigma$ scatter as a function of $v$ could be calculated. These are shown in Fig. 3 together with the original data. The solid curve is the theoretical curve (see above equation) fit to the average of the bootstrap runs. Examining Fig. 3, we find three regimes. Around the median contour in the regime $|v|<|0.5|$, we see that the data are shifted to the right relative to the theoretical curve. This suggests that we are seeing an excess of isolated low-density regions or bubblelike voids. Melott et al. (1989) have generated a toy bubble distribu- 


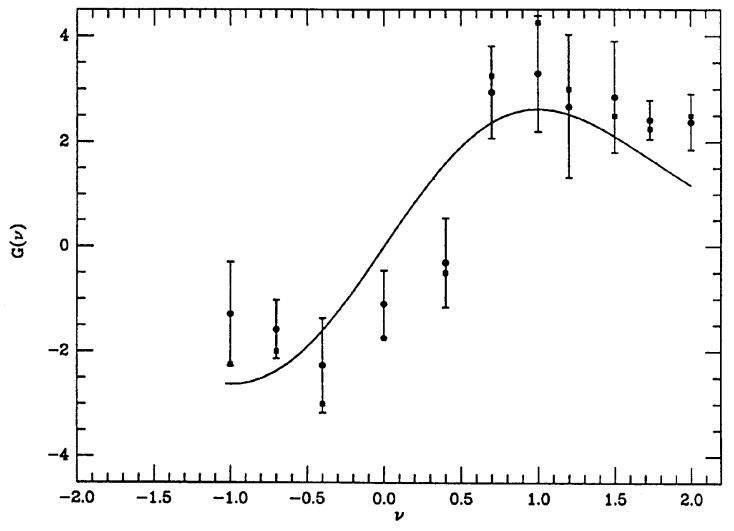

FIG. 3. The genus measured from our redshift data. Open squares are the genus from the original sample and filled dots and error bars are the mean and $1 \sigma$ variation in 12 bootstrap replacement runs. The solid curve is the theoretical curve fit to the average genus from the bookstrap runs.

tion, where particles are placed on the surfaces of irregular space-filling polyhedra. Similar shift to the right of the $g(v)$ curve is observed [see their Figs. 4(a) and 4(b) ]. In addition to the observed curve not passing through the origin, the toy model curve shows a sharper transition from positive to negative genus than the analytical gaussian curve and a slower falloff to zero at negative $v$. These two properties are also observed in our data. In the high density regime $(v>0.5)$, we find a reasonable fit to the $g(v)$ curve.

To summarize, we find that our data deviate from the predicted curve for a two-dimensional Gaussian random field in three similar ways to that of a curve produced by slices through a toy model. The toy model is generated by placing particles on the surfaces of irregular space filling polyhedra. This result suggests that we are sampling a similar sheet like distribution in our data.

\section{CONCLUSIONS}

We have investigated the galaxy distribution surrounding the Sculptor void. We have added 113 redshifts to the known redshifts in this region. To determine the nature of the topology of the galaxy distribution in this region, we have applied the topology method. The method we have applied involves calculating the genus (a topological measure) of contours of the density field. The density field is derived from the data by smoothing with a Gaussian. By comparing the observed genus for varying density contours with that expected for a gaussian field we find objective evidence for the existence of a topology of galaxies in sheets surrounded by voids.

It is a pleasure to thank Rien van de Weygaert and Adrian Melott for helpful discussions. Bob Donahue provided much help with the plotting software at NMSU. We enjoyed the able assistance of Hector Vega and Helmut Herborn at the $1.5 \mathrm{~m}$ telescope.

\section{REFERENCES}

Abell, G. O., Corwin, H. G., \& Olowin, R. P. 1989, ApJS, 70, 1

Bahcall, N., \& Soneira, R. 1983, ApJ, 270, 20

Batuski, D. J. \& Burns, J. O. 1985, ApJ, 299, 5

Bertschinger, E., \& Juszkiewicz, R. 1988, ApJ, 334, L 59

Barrow, J. D., Bhavsar, S. P., \& Sonoda, D. M. 1984, MNRAS, 210, 19p da Costa, L. N., et al. 1988, ApJ, 327, 544

da Costa, L. N., Pellegrini, P. S., Davis, M., Meiksin, A., Sargent, W. L. W., \& Tonry, J. 1991, ApJS, 75, 935

de Lapparent, V., Geller, M. J., \& Huchra, J. P. 1986, ApJ, 302, L 1

Einasto, J., Joeveer, M., \& Saar, E. 1980, MNRAS, 193, 353

Fairall, A. P. 1988, MNRAS, 230, 69

Geller, M. J., \& Huchra, J. P. 1989, Science, 246, 897

Gott, J. R., Melott, A. L., \& Dickinson, M. 1986, ApJ, 306, 341

Gott III, J. R., 1988, PASP, 100, 1307

Hamilton, A. J. S., Gott, J. R., \& Weinberg, D. H. 1986, ApJ, 309, 1

Haynes, M. P., \& Giovanelli, R. 1986, ApJ, 306, L55

Haynes, M. P., \& Giovanelli, R. 1988, in Large Scale Motions in the Universe, Pontifical Academy of Sciences Study Week, No. 27, edited by V.

Rubin and G. Coyne (Specola Vaticana, Rome), p. 31

Kaiser, N. 1988, MNRAS, 231, 149

Karachentsev, I. D., \& Kopylov, A. I. 1990, MNRAS, 243, 390

Keel, W. C. 1983, ApJ, 269, 466
Kirshner, R. P., Oemler, A., Schechter, P. L., \& Schectman, S. A. 1987, ApJ, 314, 493

Klypin, A. A., \& Kopylov, A. I. 1983, SvAL, 9, 41

Lauberts, A., \& Valentijn, E. A. 1989, The Surface Photometry Catalogue of the ESO-Uppsala Galaxies (European Southern Observatory, Garching)

Lynden-Bell, D., Faber, S. M., Davies, R. L., Dressler, A., Terlevich, R., \& Wegner, G. 1988, ApJ, 326, 19

Maddox, S. J., Efstathiou, G., Sutherland, W. J., \& Loveday, J. 1990, MNRAS, 242, 43 P

Maurellis, A., Fairall, A. P., Martravers, D. R., \& Ellis, G.F.R. 1990, A\&A, 229,75

Melott, A. L. 1990, Phys. Rep., 193,

Melott, A. L., Cohen, A. P., Hamilton, A. J. S., Gott III, J. R., \& Weinberg, D. H. 1989, ApJ, 345, 618

Ostriker, J. P., \& Suto, Y. 1990, ApJ, 348, 378

Park, C., Gott III, J. R., Melott, A. L., \& Karatchensev, I.D. 1991, preprint

Parker, Q. A., Beard, S. M., \& MacGillivray, H. T. 1987, A \& A, 173, L 5

Rhee, G. F. R. N., \& Katgert, P. 1987, A\&AS, 72, 243

Tonry, J., \& Davis, M. 1979, AJ, 84, 1511

Weinberg, D. H. 1988, PASP, 100, 1373 